\title{
Communications: A systematic method for locating transition structures of $A+B-->X$ type reactions.
}

\author{
AUTHOR(S):
}

Maeda, Satoshi; Morokuma, Keiji

\section{CITATION:}

Maeda, Satoshi ... [et al]. Communications: A systematic method for locating transition structures of A+B-->X type reactions.. The Journal of chemical physics 2010, 132(24): 241102.

ISSUE DATE:

2010-06-28

URL:

http://hdl.handle.net/2433/126689

RIGHT:

(C) 2010 American Institute of Physics 


\title{
Communications: A systematic method for locating transition structures of $A+B \rightarrow X$ type reactions
}

\author{
Satoshi Maeda ${ }^{1,2, a)}$ and Keiji Morokuma ${ }^{2,3, b)}$ \\ ${ }_{1}^{1}$ The Hakubi Center, Kyoto University, Kyoto 606-8501, Japan \\ ${ }^{2}$ Fukui Institute for Fundamental Chemistry, Kyoto University, Kyoto 606-8103, Japan \\ ${ }^{3}$ Department of Chemistry and Cherry L. Emerson Center for Scientific Computation, Emory University, \\ Atlanta, Georgia 30322, USA
}

(Received 18 May 2010; accepted 5 June 2010; published online 24 June 2010)

\begin{abstract}
Search for transition structures (TSs) as first-order saddles is one of the most important tasks in theoretical study of chemical reaction. Although automated search has been established either by starting from a local minimum (MIN) or by connecting two MINs, there is no systematic method which can locate TSs of $\mathrm{A}+\mathrm{B} \rightarrow \mathrm{X}(+\mathrm{Y})$ type reactions starting from separated reactants. We propose such an approach for the first time; it was demonstrated to work very well in the SN2, Diels-Alder, and Wittig reactions. (C) 2010 American Institute of Physics. [doi:10.1063/1.3457903]
\end{abstract}

Since the intrinsic reaction coordinate (IRC) was defined $^{1}$ as the mass-weighted steepest decent pathway (MW-SDP) starting from a transition structure (TS) and practical methods for computation of IRC were developed, ${ }^{2-4}$ reaction pathways of numerous chemical reactions have been elucidated by calculations of IRC. To calculate an IRC, one has to locate a TS on a potential energy surface (PES) as the starting point. Hence, there have been considerable efforts to develop efficient methods locating $\mathrm{TS}(\mathrm{s}) .^{5-7}$ The most fundamental tools are geometry optimization methods, ${ }^{8-10}$ which can locate one TS starting from an initial guess when the guess is appropriate.

If geometries of both reactant and product are known beforehand, one can use double-ended methods which can search for a path connecting the two geometries, e.g., the synchronous transit method, ${ }^{11}$ the self penalty walk method, ${ }^{12}$ the nudged elastic band method, ${ }^{13}$ the string method, ${ }^{14}$ the growing string method, ${ }^{15}$ and others. ${ }^{5,6}$ These methods can be used in automated samplings of many TSs when they are applied to all (or randomly selected) pairs of local minima (MINs) obtained by an automated MIN sampling. ${ }^{7}$ There are several approaches which can find out many TSs starting from single MIN: ${ }^{5,6}$ the gradient extremal following (GEF) method, ${ }^{16}$ the eigenvector following (EVF) method, ${ }^{17}$ the reduced gradient following (RGF) method, ${ }^{18}$ and the anharmonic downward distortion following (ADDF) method. ${ }^{19}$ Among these, EVF is suitable in random samplings, and it has been employed extensively in studies on conformational rearrangements and cluster structure transitions in combination with molecular mechanics force fields. ${ }^{7}$ In contrast to GEF, EVF, and RGF, all of which often may enter into regions far away from IRCs, ADDF can more efficiently follow approximate reaction pathways avoiding unimportant regions of PES. Hence, ADDF has been used in automated global reaction route mapping (GRRM) method for PESs of expensive quantum mechanical calculations. ${ }^{19,6}$

\footnotetext{
${ }^{a)}$ Electronic mail: smaeda@fukui.kyoto-u.ac.jp.

${ }^{b)}$ Electronic mail: morokuma@emory.edu.
}

Many reactions, including some organic and gas-phase reactions, fall into the $\mathrm{A}+\mathrm{B} \rightarrow \mathrm{X}(+\mathrm{Y})$ type. Since there are shallow van der Waals potential wells between $A$ and $B$ in general, the above automated methods can be applied without modification. However, such wells are not always close to TSs but are often located in very floppy regions of PESs, which prevents to identify correct reaction coordinates in automated search. Since topologies of PES involved in initial association states between A and B are very different from those in the product minimum of $\mathrm{X}$, a method specially suited for such systems should be developed. In this paper, we propose such an approach.

Let us think about pressing one reactant to the other with a constant force. Here, we propose a principle; under the force a distance between two fragments becomes minimum in the most reactive orientation. In other words, the two fragments can approach most closely to each other around an entrance of mountain pass located in such orientation. Based on this principle, the problem finding a reaction path can be replaced by a task of locating an orientation in which the distance between two fragments is minimum under the force. Then, continuous increase of the strength of force will reduce the minimum distance gradually, and finally, the product side will be reached crossing a geometry close to corresponding TS. How this principle works in actual chemical reactions will be demonstrated below.

In this study, such orientations between a pair of fragments A and B are located as local minima on the following artificial energy function, $F$ :

$$
F=E+\alpha \frac{\sum_{i \in \mathrm{A}} \Sigma_{j \in \mathrm{B}}\left[\left(R_{i}+R_{j}\right) / r_{i j}\right]^{p} r_{i j}}{\sum_{i \in \mathrm{A}} \Sigma_{j \in \mathrm{B}}\left[\left(R_{i}+R_{j}\right) / r_{i j}\right]^{p}},
$$

where $E$ is potential energy as a function of the atomic coordinates $\left\{Q_{i}\right\}, \alpha(\geq 0)$ is a parameter of magnitude of artificial attractive force, $R_{i}$ and $R_{j}$ are covalent radii of the $i$ th and $j$ th atoms, respectively, $r_{i j}$ is a distance between the $i$ th and $j$ th atoms, $p$ is an arbitrary integer explained below, and summations are taken over all pairs of atoms in the frag- 


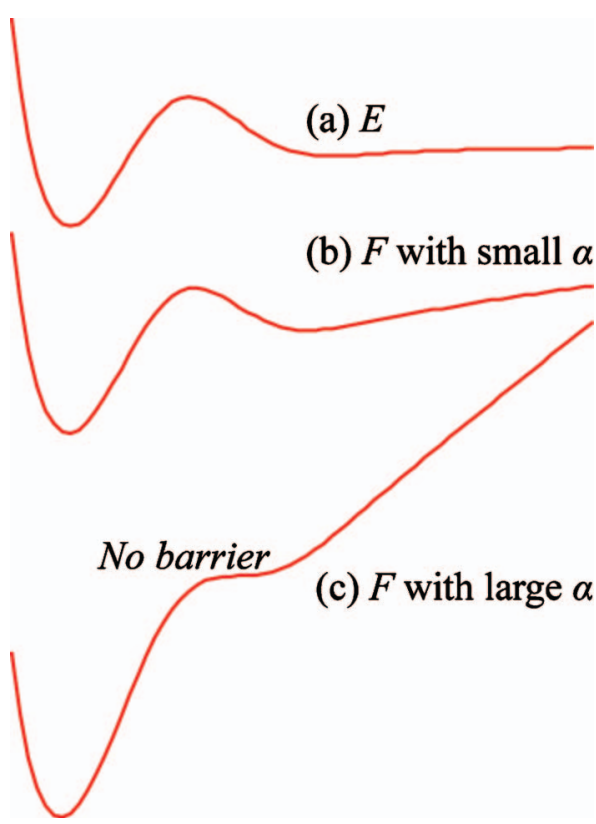

FIG. 1. (a) A one-dimensional curve of potential energy $E$, (b) a curve of $F$ in Eq. (1) with small $\alpha$, and (c) a curve of $F$ with large $\alpha$.

ments A and B. When both A and B are composed of single atom, the last term in Eq. (1) becomes just $\alpha r_{\mathrm{AB}}$, which represents a constant artificial attractive force $-\alpha$ between the atoms A and B. Figure 1 shows a typical diatomic potential curve $E$ and two curves of $F$ with different $\alpha$. At small $\alpha$ a local minimum is created before the barrier, and at sufficiently large $\alpha$ the barrier disappears and the curve is always downhill toward the minimum. In the case of general fragments including many atoms, imposing a constant force between all pairs of atoms is not suitable. This is because a part of fragments may dissociate during a reaction and the artificial force prevents the dissociations. Therefore, in Eq. (1) the artificial forces are imposed between a few pairs of atoms in a close distance by using a weight function of the modified Shepard interpolation, ${ }^{20}$ where a parameter of the weight function $p$ was set to 6 after some tests. We found that weights of $\mathrm{H}$ atom tend to be too large. Hence, the $R$ of $\mathrm{H}$ atom was set to zero in this study. The parameter $\alpha$ should be adjusted by users. Since many researchers are familiar with values of collision energy rather than force, assuming that the interatomic interaction potential is expressed by a generic Lennard-Jones 6-12 potential, one can rewrite $\alpha$ as follows:

$$
\alpha=\frac{\gamma}{\left[2^{-1 / 6}-(1+\sqrt{1+\gamma / \varepsilon})^{-1 / 6}\right] R_{0}} .
$$

This equation represents an average force acting on two atoms in the energy range $0<E<\gamma$ reflected by the LennardJones potential, where we used $R_{0}$ and $\varepsilon$ to be the values for argon clusters $\left(R_{0}=3.8164 \AA\right.$ and $\left.\varepsilon=1.0061 \mathrm{~kJ} / \mathrm{mol}\right)$. Although this transformation is not essential, it allows us to specify the parameter in units of energy, where $\gamma$ is related to collision energy on the Lennard-Jones potential. We use $\gamma$ (in $\mathrm{kJ} / \mathrm{mol}$ ) instead of $\alpha$ throughout in the following discussions.

Four well-known organic reactions shown in Fig. 2 were considered: (R1, R2) the SN2 reactions, (R3) the Diels-

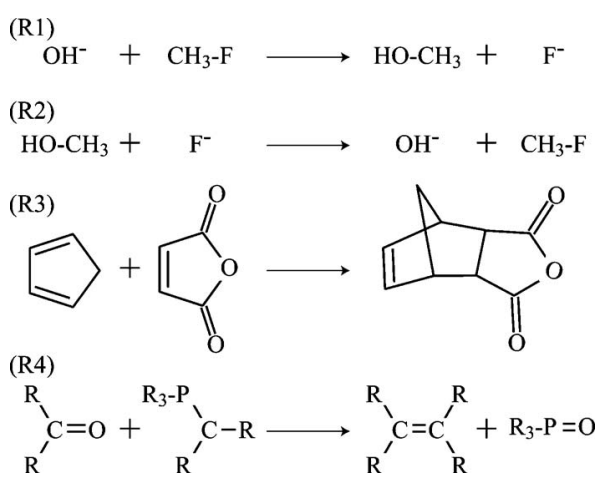

FIG. 2. The SN2 reactions (R1) and (R2), the Diels-Alder reaction (R3), and the Wittig reaction (R4).

Alder reaction, and (R4) the Wittig reaction with $\mathrm{R}=\mathrm{CH}_{3}$. Potential energy values, gradient vectors, and Hessian matrices were computed at the B3LYP/6-31+ $\mathrm{G}^{*}$ level using the GAUSSIAN09 programs. ${ }^{21}$ At small $\gamma$ (or small $\alpha$ ) a minimization of $F$ converges to a pre-reaction complex, while at large $\gamma$ a product structure will be reached. Hence, starting from $\gamma=0 \mathrm{~kJ} / \mathrm{mol}$, we increased $\gamma$ by $50 \mathrm{~kJ} / \mathrm{mol}$ until a minimization converges to a product side for each reaction. The barriers were crossed with $\gamma=100 \mathrm{~kJ} / \mathrm{mol}$ for $\mathrm{R} 1$, $200 \mathrm{~kJ} / \mathrm{mol}$ for R2, $150 \mathrm{~kJ} / \mathrm{mol}$ for R3, and $50 \mathrm{~kJ} / \mathrm{mol}$ for R4, respectively, where we denote these values as $\gamma_{0}$. Figure 3 shows optimized structures with $\gamma=0$ and $\gamma=\gamma_{0}-50 \mathrm{~kJ} / \mathrm{mol}$, except for R4 where an optimized structure with $\gamma=\gamma_{0}-25 \mathrm{~kJ} / \mathrm{mol}$ is shown because here $\gamma_{0}=50 \mathrm{~kJ} / \mathrm{mol}$. When $\gamma=0$, the distance between two fragments is very long. By imposing the forces with nonzero $\gamma$, artificial complexes with smaller interfragment distances can be obtained. The reaction R2 is an extreme example in which there is no local minimum before the TS and energy minimization on the PES always converged to R2-1. However, one can locate a complex R2-2 near the TS as a minimum on the energy $F$ of Eq. (1).

Figure 4(a) shows the IRC profile on $E$ of reactions R1 and $\mathrm{R} 2$, where $\mathrm{R} 2$ is the inverse reaction of R1. Starting from the common TS, R1-1 and R2-1 in Fig. 3 were reached in the backward and forward IRC followings, respectively. This profile is consistent with the one calculated by the published results although a different electronic structure method was used. $^{22}$ Then, starting from R1-1, a MW-SDP was computed on $F$ with $\gamma=\gamma_{0}(100 \mathrm{~kJ} / \mathrm{mol})$, in which 133 gradients and 2 Hessians were required. Its profile is shown in Fig. 4(b) with a blue dashed curve. As seen in this figure, the curve is always downhill and the MW-SDP reached the product side. Meanwhile, a profile of potential energy $E$ along this MWSDP, shown in Fig. 4(b) with a red solid curve, has a peak corresponding to an approximate barrier of this reaction. A structure at the highest energy point along this profile is shown in Fig. 4(b), where a structure of the true TS in black body is overlapped behind the structure. The highest energy structure is very similar to the true TS, and a TS optimization starting from this approximate structure converged after 13 optimization steps, where exact Hessian was computed once at the first point and default options in GAUSSIAN09 were employed, i.e., $\mathrm{Opt}=(\mathrm{TS}, \mathrm{CalcFC}){ }^{21}$ In the case of $\mathrm{R} 2$, a 
(R1-1)

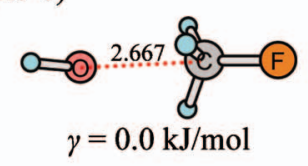

(R2-1)
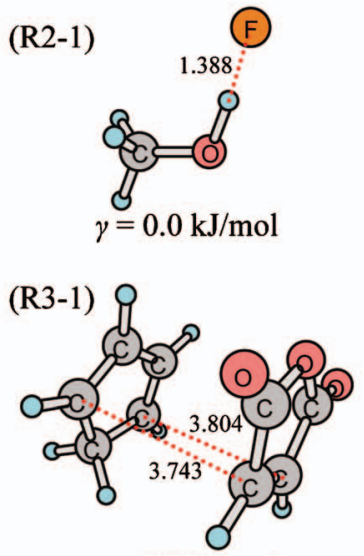

$\gamma=0.0 \mathrm{~kJ} / \mathrm{mol}$

(R4-1)

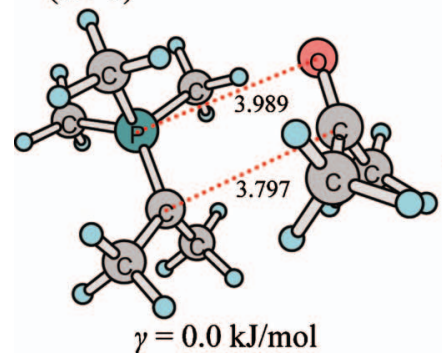

(R1-2)

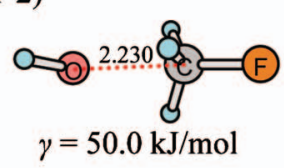

(R2-2)

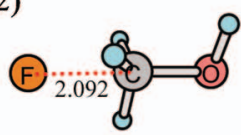

$\gamma=150.0 \mathrm{~kJ} / \mathrm{mol}$
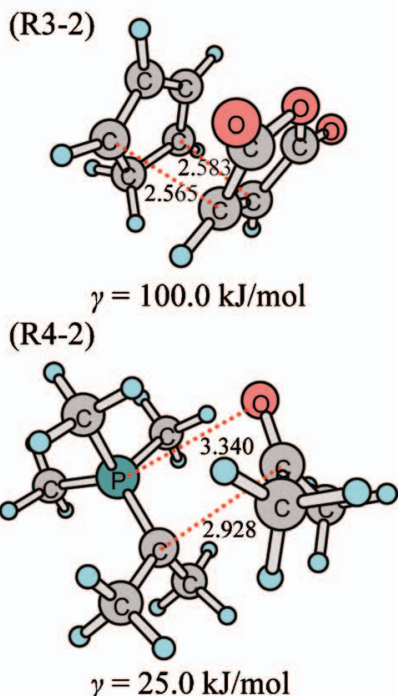

FIG. 3. Weakly bound pre-reaction complexes (R1-1, R2-1, R3-1, and R4-1) and reactive collision complexes (R1-2, R2-2, R3-2, and R4-2) pressed by artificial forces in Eq. (1), where $\gamma$ corresponds to (model) collision energy in Eq. (2).

MW-SDP with $\gamma=\gamma_{0}(200 \mathrm{~kJ} / \mathrm{mol})$ shown in Fig. 4(c) was obtained starting from a point marked by along the IRC profile in Fig. 4(a), where in the MW-SDP calculation 90 gradients and 2 Hessians were computed. A structure at the top of potential energy profile in Fig. 4(c) is also similar to the true TS, and the TS optimization starting from this structure converged after 18 steps.

To find other possible channels systematically, we performed optimizations starting from random orientations for $\mathrm{R} 1$ and $\mathrm{R} 2$ at $\gamma=100 \mathrm{~kJ} / \mathrm{mol}$, where generation of random orientation was terminated when last ten optimizations converged to the structures which were already found in earlier optimizations. This procedure gave three local minima for R1: a complex $\mathrm{CH}_{3} \mathrm{OH} \cdots \mathrm{F}^{-}$which is a product of R1, i.e., R2-1 like structure, a complex $\mathrm{CH}_{2} \mathrm{~F}^{-} \cdots \mathrm{HOH}$ which is a product of $\mathrm{OH}^{-}+\mathrm{CH}_{3} \mathrm{~F} \rightarrow \mathrm{CH}_{2} \mathrm{~F}^{-}+\mathrm{H}_{2} \mathrm{O}$ reaction, and a complex $\mathrm{OH}^{-} \cdots \mathrm{FCH}_{3}$ which was converged to $\mathrm{CH}_{3}{ }^{-}+\mathrm{HOF}$ at high $\gamma$. Three local minima were found also in the case of R2: the precursor of $\mathrm{SN} 2$ reaction, i.e., R2-2 like structure, a complex $\mathrm{CH}_{3} \mathrm{O}^{-} \cdots \mathrm{HF}$ which is a product of $\mathrm{F}^{-}+\mathrm{CH}_{3} \mathrm{OH}$ $\rightarrow \mathrm{CH}_{3} \mathrm{O}^{-}+\mathrm{HF}$ reaction, and a complex $\mathrm{CH}_{2} \mathrm{OH}^{-} \cdots \mathrm{HF}$ which is a product of $\mathrm{F}^{-}+\mathrm{CH}_{3} \mathrm{OH} \rightarrow \mathrm{CH}_{2} \mathrm{OH}^{-}+\mathrm{HF}$ reaction. In these procedures, 1943 gradient vectors and 49 Hessian matrices were computed in R1 and 1355 gradients and 39 Hessians were required in R2. As shown above and below,

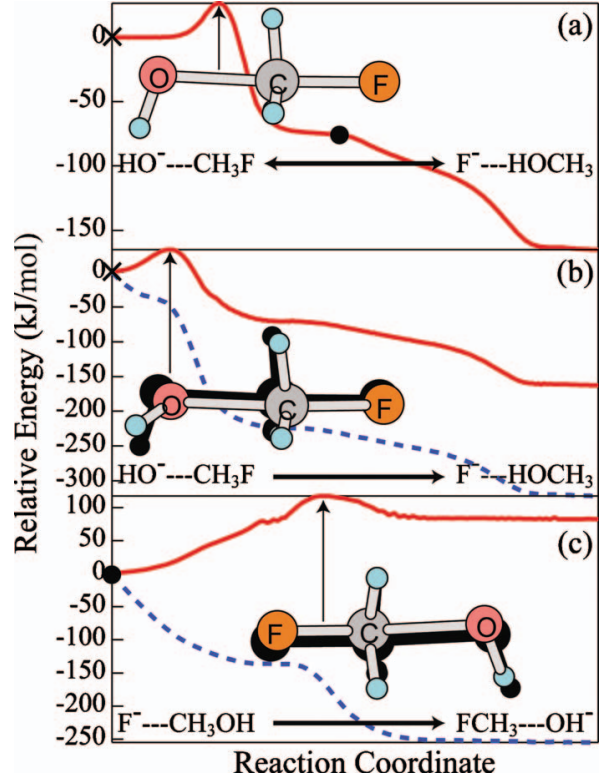

FIG. 4. (a) The IRC profile (on $E$ ) of SN2 reactions (R1) and (R2), (b) a profile of MW-SDP on $F$ in Eq. (1) with $\gamma=100 \mathrm{~kJ} / \mathrm{mol}$ starting from the geometry (R1-1) marked by $\times$ in (a) (blue dashed curve) and a profile of potential energy $E$ along the MW-SDP on $F$ (red solid curve), and (c) a profile of MW-SDP on $F$ with $\gamma=200 \mathrm{~kJ} / \mathrm{mol}$ starting from the geometry marked by in (a) (blue dashed curve) and a profile of potential energy along the MW-SDP (red solid curve). In (b) and (c), geometries corresponding to the highest energy points along solid curves (artificial TS structures as guesses of the true TS) are shown, where the true TS in black body is overlapped behind them for comparison.

100-200 gradients and a few Hessians are further required per path in the MW-SDP and true TS calculations. For comparison, we performed automated searches using the GRRM method. The GRRM searches were started from R1-1 and R2-1 with the standard (full-ADDF) option, ${ }^{19}$ where pathways from other MINs were not considered. Although the GRRM method was able to find the TS of R1, it failed to leach the TS of R2. This is because the TS is too far to be searched from R2-1. Moreover, the GRRM searches required $25758 / 747$ gradient/Hessian and $24230 / 653$ gradient/ Hessian computations from R1-1 and R2-1, respectively, which are much more than those of the present method. This is because the GRRM gave many intrafragment reaction channels such as methyl group rotations, $\mathrm{H}$ atom dissociations, $\mathrm{H}_{2}$ dissociations, and others. Such intrafragment pathways should be searched by applying a method to each fragment separately for best efficiency. We do not claim that the present method is overall superior to minimum-to-minimum methods including the GRRM method, since it does not work in isomerization type reactions at all. We suggest use of different methods in different purposes, and the present method is the only approach specially suited for $\mathrm{A}+\mathrm{B} \rightarrow \mathrm{X}$ type reactions hitherto.

Figure 5(a) shows the IRC profile of Diels-Alder reaction. A MW-SDP on $F$ with $\gamma=\gamma_{0}(150 \mathrm{~kJ} / \mathrm{mol})$ gave a reasonable approximate structure of TS as the highest energy point of potential energy profile, as shown in Fig. 5(b), where the TS optimization starting from this structure converged after nine steps and 103 gradients and 2 Hessians were computed in the MW-SDP integration. Figure 5(c) 


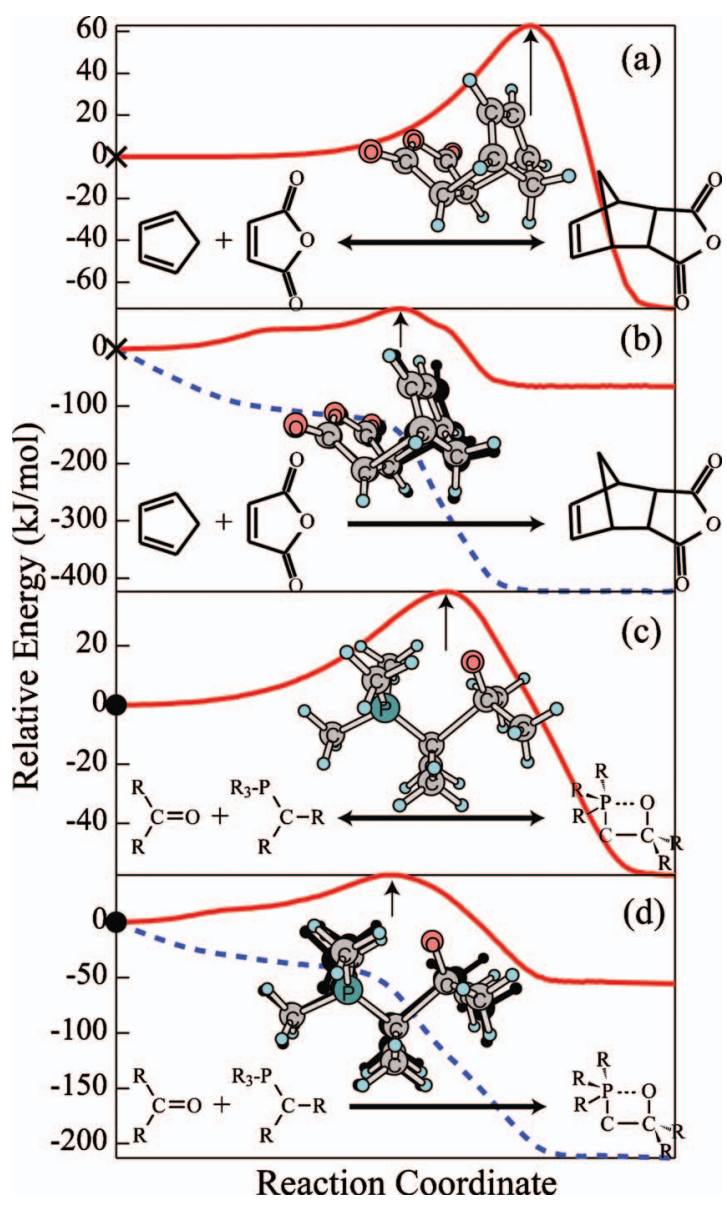

FIG. 5. (a) The IRC profile of Diels-Alder reaction, R3, (b) a profile of MW-SDP on $F$ in Eq. (1) with $\gamma=150 \mathrm{~kJ} / \mathrm{mol}$ starting from the geometry marked by $X$ in (a) (blue dashed curve) and a profile of potential energy along the MW-SDP (red solid curve), (c) the IRC profile of Wittig reaction (R4), and (d) a profile of MW-SDP on $F$ with $\gamma=50 \mathrm{~kJ} / \mathrm{mol}$ starting from the geometry marked by $\bullet$ in (c) (blue dashed curve) and a profile of potential energy along the MW-SDP (red solid curve). In (a) and (c), geometries of true TS are shown, while in (b) and (d), geometries corresponding to the highest energy points along solid curves (artificial structures as guesses of the true TSs) are shown, where the true TSs in black body are overlapped behind them for comparison.

shows the IRC profile of Wittig reaction. Although this reaction is a multistep reaction, ${ }^{23}$ the second and latter steps are not considered in this study since they are not the $\mathrm{A}+\mathrm{B} \rightarrow \mathrm{X}$ type and which should be treated by minimum-tominimum methods. A MW-SDP on $F$ with $\gamma=\gamma_{0}(50 \mathrm{~kJ} / \mathrm{mol})$ again provided a reasonable guess of TS as the highest energy point of potential profile, as shown in Fig. 5(d), where the TS optimization starting from this structure converged after ten steps and 148 gradients and 2 Hessians were required in the MW-SDP calculation.

In summary, an efficient algorithm to obtain a TS of $\mathrm{A}+\mathrm{B} \rightarrow \mathrm{X}$ type reaction is as follows: (1) determination of (approximate) $\gamma_{0}$ by performing minimizations of $F$ with systematically changing the parameter $\gamma,(2)$ a computation of MW-SDP on $F$ with $\gamma=\gamma_{0}$, and (3) a TS optimization starting from the highest energy point of potential energy profile along the MW-SDP by using a standard TS optimiza- tion method. ${ }^{8-10}$ The first step can be done either by starting from many random orientations or by starting from a guessed orientation. The latter is faster if there is a very good guess, while the former will give a systematic result including many possible channels. It should be emphasized that there is no physical meaning on structures on the function $F$ of Eq. (1); we search for and use such artificial structures on $F$ as guesses of true TSs on $E$.

There have been a number of studies on relationships between chemical reactivity and interparticle interaction represented by Fukui's frontier orbital theory ${ }^{24}$ and the Woodward-Hoffmann rules. ${ }^{25}$ Although such studies undoubtedly have great values, none of them has an ability to systematically predict geometries of TS on quantum chemical PESs. The present method can be used in automated prediction of unknown reaction pathways when energy minimizations on $F$ in Eq. (1) are performed starting from many random orientations. Moreover, an extension to reactions involving three or more particles is straightforward. Further applications of the present method will be reported in forthcoming papers.

The authors thank Professor Masanobu Uchiyama for suggesting difficulties in finding transition states in some $\mathrm{A}+\mathrm{B} \rightarrow \mathrm{X}$ type organic reactions. This work is partly supported by a grant from Japan Science and Technology Agency with a Core Research for Evolutional Science and Technology (CREST) in the Area of High Performance Computing for Multiscale and Multiphysics Phenomena as well as a grant from AFOSR (Grant No. FA9550-07-1-0395).

${ }^{1}$ K. Fukui, J. Phys. Chem. 74, 4161 (1970).

${ }^{2}$ K. Ishida, K. Morokuma, and A. Komornicki, J. Chem. Phys. 66, 2153 (1977).

${ }^{3}$ M. Page and J. W. McIver, Jr., J. Chem. Phys. 88, 922 (1988).

${ }^{4}$ C. Gonzalez and H. B. Schlegel, J. Chem. Phys. 90, 2154 (1989).

${ }^{5}$ H. B. Schlegel, J. Comput. Chem. 24, 1514 (2003).

${ }^{6} \mathrm{~F}$. Jensen, Introduction to Computational Chemistry, 2nd ed. (Wiley, Chichester, 2007).

${ }^{7}$ D. J. Wales, Int. Rev. Phys. Chem. 25, 237 (2006).

${ }^{8}$ H. B. Schlegel, J. Comput. Chem. 3, 214 (1982).

${ }^{9}$ P. Császár and P. Pulay, J. Mol. Struct. 114, 31 (1984).

${ }^{10}$ A. Banerjee, N. Adams, J. Simons, and R. Shepard, J. Phys. Chem. 89, 52 (1985).

${ }^{11}$ T. A. Halgren and W. N. Lipscomb, Chem. Phys. Lett. 49, 225 (1977).

${ }^{12}$ R. Elber and M. Karplus, Chem. Phys. Lett. 139, 375 (1987).

${ }^{13}$ G. Henkelman, B. P. Uberuaga, and H. Jónsson, J. Chem. Phys. 113, 9901 (2000).

${ }^{14}$ W. E, W. Ren, and E. Vanden-Eijnden, Phys. Rev. B 66, 052301 (2002).

${ }^{15}$ B. Peters, A. Heyden, A. T. Bell, and A. Chakraborty, J. Chem. Phys. 120, 7877 (2004).

${ }^{16}$ J.-Q. Sun and K. Ruedenberg, J. Chem. Phys. 98, 9707 (1993).

${ }^{17}$ C. J. Cerjan and W. H. Miller, J. Chem. Phys. 75, 2800 (1981).

${ }^{18}$ W. Quapp, M. Hirsch, O. Imig, and D. Heidrich, J. Comput. Chem. 19, 1087 (1998)

${ }^{19}$ K. Ohno and S. Maeda, Phys. Scr. 78, 058122 (2008).

${ }^{20}$ M. A. Collins, Theor. Chem. Acc. 108, 313 (2002).

${ }^{21}$ M. J. Frisch, G. W. Trucks, H. B. Schlegel et al., GAUSSIAN 09, Revision A.1, Gaussian, Inc., Wallingford, CT, 2009.

${ }^{22}$ L. Sun, K. Song, and W. L. Hase, Science 296, 875 (2002).

${ }^{23}$ R. Robiette, J. Richardson, V. K. Aggarwal, and J. N. Harvey, J. Am. Chem. Soc. 128, 2394 (2006).

${ }^{24}$ K. Fukui, T. Yonezawa, and H. Shingu, J. Chem. Phys. 20, 722 (1952).

${ }^{25}$ R. B. Woodward and R. Hoffmann, J. Am. Chem. Soc. 87, 395 (1965). 